\title{
Burnout em estudantes de graduação em Odontologia
}

\author{
Camila Prado das Neves*, Dayane Machado Ribeiro**
}

\section{RESUMO}

A síndrome de Burnout (SB) é uma condição crônica multidimensional. Observa-se que um número significativo de estudantes e profissionais da área da saúde, especialmente da Odontologia, tem sido acometido por este agravo. O presente estudo objetivou verificar a prevalência da SB entre os estudantes de graduação em Odontologia de $1^{\mathrm{a}}, 5^{\mathrm{a}}$ e $10^{\mathrm{a}}$ fases da UFSC. O instrumento de coleta de dados foi um questionário sobre dados demográficos e econômicos, perfil do estudante e o Inventário de Burnout Maslach - Student Survey (MBI-SS). A prevalência da SB foi de $10,89 \%$. Houve associação estatisticamente significativa entre as fases do curso e níveis percepção da estrutura física da instituição de ensino e pensamento de desistir do curso. Também entre a ocorrência da SB e fase do curso, horas semanais de lazer, horas diárias de sono, necessidade de professor particular, percepção do próprio desempenho acadêmico, do desempenho dos professores e da estrutura física da instituição de ensino, consumo de medicamentos e pensamento de desistir do curso. Conclui-se que a SB foi prevalente em $10,89 \%$ dos estudantes pesquisados.

Descritores: Burnout. Estresse. Estudantes de Odontologia.
Estudante do Curso de Graduação em Odontologia da Universidade Federal de Santa Catarina (UFSC)

** Professora do Departamento de Odontologia da Universidade Federal de Santa Catarina (UFSC)

\section{INTRODUÇÃO}

O termo Burnout, do inglês, significa “combustão completa", e é usado para se referir ao transtorno crônico-adaptativo de estresse relacionado ao trabalho, a chamada Síndrome de Burnout (SB). ${ }^{1}$

A SB é uma condição crônica multidimensional, cujas principais dimensões são: exaustão emocional (EE), desumanização / despersonalização / descrença (DE) e reduzida realização no trabalho / reduzida eficácia profissional (EP). ${ }^{2}$

A SB traz consequências sobre a saúde do indivíduo por ela acometido, como, por exemplo, alterações orgânicas nos níveis do hormônio cortisol e prejuízo no desempenho das atividades laborais. ${ }^{3}$

O consumo de medicamentos devido a atividades laborais, incluindo o estudo, a percepção negativa do ambiente de trabalho e insatisfação com a atividade exercida estão relacionadas à ocorrência da $\mathrm{SB} .{ }^{4}$

Observa-se que um número significativo de estudantes e profissionais da área da saúde e, especialmente, da Odontologia, tem sido acometido pela SB..$^{5,6}$ 
A carga horária atual do curso de graduação em Odontologia se apresenta sobrecarregada, já que, para atender a demanda do mercado atual, as Diretrizes Curriculares Nacionais (DCN) determinam a formação do cirurgião-dentista como um profissional generalista e a inclusão de disciplinas das ciências sociais não isenta a formação técnica do profissional. ${ }^{7,8}$

De modo geral, os estudos disponíveis que verificam a ocorrência da $\mathrm{SB}$ em estudantes de Odontologia, e também de outras áreas, propõem a investigação e o monitoramento da SB desde o início da graduação, pois sua detecção precoce é interessante para planejar e executar a prevenção e o enfrentamento deste agravo. ${ }^{9-14}$

Dessa forma, este trabalho se propõe a verificar a prevalência da SB em estudantes do curso de graduação em Odontologia da Universidade Federal de Santa Catarina (UFSC).

\section{MÉTODOS}

Esta pesquisa caracterizou-se por um estudo transversal, de caráter descritivo, cujo método utilizado consiste em análise e interpretação dos dados coletados a partir da observação de fenômenos e causas.

O projeto foi submetido ao Comitê de Ética em Pesquisa em Seres Humanos da UFSC e aprovado sob o parecer 358.029.

A população do estudo foi composta por estudantes do curso de graduação em Odontologia da UFSC da $1^{\mathrm{a}}$., $5^{\mathrm{a}}$. e $10^{\mathrm{a}}$. fases, regularmente matriculados no semestre 2013/02.

Todos os estudantes que concordaram em participar da pesquisa, mediante a leitura e assinatura do Termo de Consentimento Livre e Esclarecido foram incluídos.

O instrumento de coleta de dados foi um questionário aplicado de forma direta em sala de aula em um momento previamente combinado com o professor responsável.

O questionário foi elaborado em uma linguagem supostamente compatível com o público-alvo e abordou questões sobre dados demográficos e econômicos (com base no critério adaptado de Classificação Econômica Brasil 2013) ${ }^{15}$, perfil do estudante (hábitos pessoais e rotina acadêmica) e avaliação da SB (através do Inventário de Burnout Maslach - Student Survey (MBI-SS) na versão em língua portuguesa). ${ }^{16}$

As variáveis pesquisadas em relação ao perfil do estudante e as opções de respostas constam do quatro 1 .

Os dados foram organizados e apurados para todas variáveis de estudo com o auxílio dos programas EpiData Entry $3.0 \mathrm{e}$ STATA 11.2

Como ponto de corte para determinação da Exaustão e Descrença utilizou-se o percentil 66 (P66) e para Eficácia Profissional o percentil 33 (P33) conforme proposta de Maslach e Jackson. ${ }^{17}$ Caracterizou-se como acometido pela SB o estudante que apresentou simultaneamente valores médios acima do P66 para Exaustão e Descrença e abaixo do P33 para Eficácia Profissional.

Para o estudo de associação, utilizouse o teste exato de Fischer, considerando-se o nível de significância $\mathrm{p} \leq 0,05$ para tomada de decisão. 
Quadro 1. Variáveis pesquisadas e respostas possíveis.

Carga horária semanal, considerando atividades teóricas, práticas e teórico-práticas: ( ) Até 15 horas-aula ( ) Entre 15 e 30 horas-aula ( ) Mais de 30 horas-aula

Participação em alguma atividade extracurricular ( ) Sim ( ) Não

Número de refeições por dia ( ) Até 1 ( ) 2 a 3 ( ) > 3

Tipo de refeição ( ) Tradicional (desjejum, almoço e jantar) ( ) Lanche

Horas semanais de lazer ( ) Nenhuma ( ) 1-5h ( ) $>5 \mathrm{~h}$

Horas diárias de sono ( ) < 6h ( ) 6-8h ( ) > 8h;

Atividade física ( ) Não ( ) Sim, horas por semana

Última visita ao médico ( ) Há menos de 1 ano ( ) Entre 2-5 anos ( ) Há mais de 5 anos

Necessidade de professor particular ( ) Sim ( ) Não

Opção do curso no vestibular ( ) $\mathbf{1}^{\mathrm{a}}$ ( ) $\mathbf{2}^{\mathrm{a}}$ ( ) $\geq 3^{\mathrm{a}}$

Expectativas do curso ( ) Péssimas ( ) Ruins ( ) Regulares ( ) oas ( ) Excelentes

Autopercepção do desempenho acadêmico ( ) Péssima ( ) Ruim ( ) Regular ( ) Boa ( ) Excelente

Percepção do desempenho docente ( ) Péssima ( ) Ruim ( ) Regular ( ) Boa ( ) Excelente

Percepção da estrutura física da instituição ( ) Péssima ( ) Ruim ( ) Regular ( ) Boa ( ) Excelente

Com quem vive ( ) Sozinho ( ) Família ( ) Amigos, colegas ( ) Outros, quem?

Tempo de deslocamento de casa até a universidade ( ) Menos de $30 \mathrm{~min}$ ( ) 30min a $1 \mathrm{~h}$ ( ) Mais de $1 \mathrm{~h}$

Quem financia os estudos ( ) Família ( ) Bolsa estudantil ( ) Outros, quem?

Frequência de encontro com a família ( ) Todos os dias, ( ) Mensalmente ( ) Semestralmente

( ) Anualmente ( ) Fica mais de 1 ano sem ver a família

Doença sistêmica ( ) Sim, qual? _ ( ) Não

Familiar com doença sistêmica ( ) Sim, qual?

Uso de medicamentos devido aos estudos ( ) Nunca ( ) Às vezes, qual?

( ) Com frequência, qual?

Pensamento em desistir do curso ( ) Nunca ( ) Às vezes ( ) Com frequência

\section{RESULTADOS E DISCUSSÃO}

Até o final do $2 .^{\circ}$ mês de aula do semestre 2013/02, o total de estudantes matriculados na 1. ${ }^{\mathrm{a}}, 5$. $^{\mathrm{a}}$ e $10{ }^{\mathrm{a}}$ fases do curso de graduação em Odontologia na UFSC foi, respectivamente 57,41 e 30 . Isso totalizou em 128 estudantes convidados a participar da pesquisa. O número (percentual) de estudantes que aceitaram participar da pesquisa de cada fase foi: 45 (78,95\%), $33(80,49 \%)$ e 27 (90\%) respectivamente. Observa-se um decréscimo no número absoluto de estudantes, à medida que o curso avança, como ocorre na universidade de Huesca e ao contrário do que ocorre na universidade de Santiago de Compostela, em 2010. ${ }^{18}$

No total, 105 estudantes participaram deste estudo, correspondendo a uma taxa de resposta total de $82,03 \%$. Este valor é semelhante ao encontrado nos trabalhos de Campos et al. $(2012)^{4}$, onde a taxa de resposta foi de $78,3 \%$ e de Montero-Marin et al. $(2011)^{18}$, que teve $83,07 \%$ de resposta total.

Os estudantes da $1 .^{\mathrm{a}}$ fase correspondem a $42,86 \%$ da amostra, enquanto os da $5 .^{\text {a }}$ fase a $31,43 \%$ e os da $10 .^{a}$ a $25,71 \%$. A idade dos participantes variou de 17 a 28 anos, sendo 19 anos a idade que mais se repetiu (moda). Comparativamente, os estudantes de Odontologia de Huesca e Santiago de Compostela tinham idade que variavam entre 18 e 41 anos. ${ }^{18}$ Apesar de a faixa de idade dos estudantes de Odontologia europeus ser maior que a dos brasileiros, a média de idade nestas universidades foi de 22,05 anos $(\mathrm{DP}=3,57)^{18}$, se assemelhando à do 
presente estudo, onde encontrou-se 21,25 anos $(\mathrm{DP}=2,53)$ como idade média dos participantes.

A maioria dos estudantes é do sexo feminino (67\%). A feminilização dos cursos de Odontologia é uma tendência já observada nas universidades em todo o mundo., ${ }^{4,-10,13-}$ 14,18-19 Existem estudos demonstrando o predomínio de estudantes do sexo masculino nos cursos de Odontologia, porém isso ocorre ainda no final do século XX. ${ }^{19-21}$ Há, contudo, pesquisas apresentando per-centuais similares entre estudantes homens e mulheres. ${ }^{11-12,22}$

A maioria da amostra é solteira (97\%). Esta é uma condição comum aos estudantes que participaram de pesquisas semelhantes realizadas na América Latina e na Europa. ${ }^{11,18}$

A maior parte dos estudantes procede do interior de Santa Catarina (47\%) e mora na capital catarinense há pelo menos 1 ano $(80 \%$ do grupo anterior). A classificação econômica dos participantes variou de A1 a D e, para apresentação dos resultados, foram estratificadas da seguinte forma: A (A1 e A2), B (B1 e B2), C e D (C1, C2 e D). A maioria dos estudantes $(47 \%)$ pertence à classe $B$, cuja renda familiar bruta varia de $\mathrm{R} \$ 2.654,00$ a $\mathrm{R} \$ 5.241,00$.

O maior número de estudantes desloca-se para a faculdade a pé $(51 \%)$ e leva menos de 30 minutos para ir de sua casa à universidade (67\%). Segundo Schmitt et al. (2013), o tráfego congestionado da capital catarinense é um problema para a sociedade. ${ }^{21}$ Assim, o fato de os estudantes morarem próximos à universidade minimiza a ocorrência de possíveis impactos negativos sobre sua saúde mental pois, para Michal (1997), o tráfego intenso e caótico é considerado um agente causador de estresse. ${ }^{23}$

A maioria dos estudantes faz mais de 3 refeições por dia (59\%), sendo estas refeições tradicionais - café da manhã, almoço e jantar (88\%). Este fator é positivo, visto que a alimentação insuficiente ou inadequada é considerada também um agente causador de estresse. $^{23}$

Cerca de metade dos participantes dedica entre 1 e 5 horas semanais para o lazer (51\%). Isso significa menos de 1 hora diária de lazer. Lemos $(2003)^{24}$ e $(2004)^{8}$ discutem a influência da carga horária exaustiva do curso de Odontologia no impedimento do estudante realizar atividades de lazer, cultura e mesmo atividades de estudo que não sejam aulas prontas. Além da carga horária registrada no currículo do curso já se apresentar pesada, o estudante de Odontologia da UFSC ainda necessita dedicar tempo extracurricular para o estudo dos conteúdos ministrados, preparação de trabalhos e seminários, organização dos planos de tratamento dos pacientes atendidos nas clínicas, compra, lavagem e esterilização de materiais, trabalhos protéticos de laboratório e além dos trabalhos de conclusão de curso, quando os estudantes estão cursando as fases finais. Fosse levado em conta que é uma necessidade do estudante o tempo para repouso, lazer e convívio social e se repensaria o currículo atual do curso de graduação em Odontologia.

Aproximadamente $\quad 65 \%$ dos estudantes dormem entre 6 e 8 horas por dia. Entretanto, aproximadamente $35 \%$ dorme menos de 6 horas diariamente. Fernandes (2006) afirma que a maioria dos adultos não se sente completamente refeito com menos de 7 horas de sono diárias. ${ }^{25}$ Assim, a redução do tempo ideal de repouso dessa parcela da amostra muito provavelmente reflete no seu desempenho acadêmico, tornando-os consequentemente mais suscetíveis à SB.

Grande parte dos estudantes (47\%) é sedentária, ou seja, não pratica nenhuma hora 
semanal de atividade física. Segundo Regis Filho e Ribeiro (2007), a atividade física, principalmente a aeróbica, é uma aliada importante no aumento da autoestima, na melhoria do sistema imune e na prevenção de doenças em geral, especialmente os distúrbios relacionados ao estresse. ${ }^{26}$ Portanto, deve-se investigar a causa do sedentarismo entre esses estudantes, para que o risco de exposição a essas doenças seja diminuído.

Cerca de metade dos estudantes $(47 \%)$ mora com a família, diferentemente dos estudantes de Odontologia da UNESPAraraquara, e da universidade de Huesca, onde a maior parte mora com amigos. ${ }^{4,18}$ Essas informações também diferem dos estudantes de Odontologia da UNESPAraçatuba, dentre os quais a maioria mora em república estudantil. ${ }^{13}$

A maioria dos participantes (54\%) cursa entre 16 e 30 horas-aula por semana e não participa de atividades extracurriculares (69\%). No entanto, $43 \%$ dos estudantes cursam mais de 30 horas-aula por semana, o que pode sugerir uma justificativa para a não aderência a atividades extracurriculares, segundo Lemos $(2003)^{24}$ e (2004) $)^{8}$. Um estudo feito em duas universidades espanholas revela que um quarto dos estudantes de Odontologia cursa entre 30 e 40 horas-aula e um terço cursa mais de 40 horas-aula. ${ }^{18}$

A maior parte dos estudantes escolheu o curso de Odontologia como primeira opção no vestibular (94\%), não teve necessidade de professor particular durante o curso (97\%), teve boas expectativas em relação ao curso (57\%), boa percepção de seu próprio desempenho acadêmico e também dos professores (59\%), considerou a estrutura física da instituição de ensino em condições regulares a péssimas (34\%), tem seus estudos financiados pelos pais (94\%), não possui doença sistêmica (84\%) e não consome medicamentos devido aos estudos (66\%). Entretanto, cerca de $35 \%$ consome algum tipo de medicamento devido aos estudos, sendo os analgésicos os medicamentos mais citados.

O estudo de Montero-Marin (2011) mostra que a maior parte dos estudantes das Universidade de Huesca e Santiago de Compostela não recebe bolsa estudantil, tendo, também, seus estudos financiados pela família e podendo se dedicar aos estudos, por não precisar trabalhar. ${ }^{18}$

A maioria dos participantes $(55 \%)$ também nunca pensou em desistir do curso. Contudo, cerca de $45 \%$ teve pelo menos $1 \mathrm{vez}$ esse pensamento, como ocorrido no estudo de Campos et al (2012). ${ }^{4}$

A prevalência da SB na amostra é de 10,89\% (IC95\% = 4,71 - 17,07). Na UNESP Araraquara, em 2009, a prevalência foi de $17 \%\left(\mathrm{IC}_{95 \%}=13,0-21,0\right)$ e pode ser considerada alta. ${ }^{4}$ Em ambos os estudos, considera-se a possibilidade de ter havido um viés de seleção, já que a participação nas pesquisas foi voluntária, podendo ter acontecido que os estudantes mais afetados não tenham participado. Os estudantes participantes do estudo de Garbin et al. (2012) não apresentaram SB, apesar de terem apresentado um nível considerado da dimensão exaustão (EE) ${ }^{13}$

É alarmante o fato de mais de $10 \%$ dos jovens estudantes pesquisados estarem sendo acometidos por este agravo crônico. Isso merece a atenção das universidades para que desenvolvam estratégias a fim de minimizar o impacto dos fatores de risco para a SB, destacando-se a carga horária sobrecarregada. A prevenção da SB evita a formação de profissionais com mais dificuldades de bem exercerem sua profissão - neste caso como cirurgiões dentistas - já que 
a SB é um agravo crônico, exigindo, portanto, tratamento por toda a vida. Da mesma forma, chama a atenção o fato de pessoas jovens estarem sendo acometidas pela SB, uma vez que doenças crônicas estão geralmente associadas à idade avançada.

$\mathrm{Na}$ Tabela 1 encontra-se o estudo de associação entre a fase do curso e a percepção dos estudantes frente à estrutura física da instituição de ensino e a ocorrência de SB.

Tabela 1 - Estudo de associação entre perfil dos estudantes e fases do curso (quando $\mathrm{p} \leq 0,05$ ). Curso de graduação em Odontologia. UFSC. Florianópolis-SC, 2013.

\begin{tabular}{lcccc} 
Característica & \multicolumn{3}{c}{ Fases do curso n (\%) } & p \\
\hline $\begin{array}{l}\text { Percepção das condições da estrutura } \\
\text { física }\end{array}$ & $\mathbf{1}^{\mathbf{a}}$ & $\mathbf{5}^{\mathbf{a}}$ & $\mathbf{1 0}^{\mathbf{a}}$ & \\
Ruins/Péssimas & $3(6,67)$ & $28(84,85)$ & $18(66,66)$ & \\
Regulares & $23(51,11)$ & $5(15,15)$ & $8(29,63)$ & \\
Boas/Excelentes & $19(42,22)$ & $-(-)$ & $1(3,7)$ & $\mathbf{0 , 0 0 0}$ \\
Já pensou em desistir do curso & & & & \\
Nunca & $36(80)$ & $12(36,36)$ & $10(37,04)$ & \\
Âs vezes & $8(17,78)$ & $17(51,52)$ & $13(48,15)$ & \\
Com frequência & $1(2,22)$ & $4(12,12)$ & $4(14,81)$ & $\mathbf{0 , 0 0 0}$ \\
Acometido pela SB* & & & & \\
Não & $100(100)$ & $26(78,79)$ & $20(83,33)$ & \\
Sim & $0(0)$ & $7(21,21)$ & $4(16,67)$ & $\mathbf{0 , 0 0 2}$ \\
\hline * SB = Síndrome de Burnout & & & &
\end{tabular}

Houve associação estatisticamente significativa entre as diferentes fases do curso pesquisadas e as variáveis: percepção da condição da estrutura física da instituição de ensino $(\mathrm{p}<0,01)$, pensamento de desistir do curso $(\mathrm{p}<0,01)$ e ocorrência da SB $(\mathrm{p}<0,05)$.

Os que menos apreciaram as condições da estrutura física da instituição de ensino foram os estudantes da $5^{\mathrm{a}}$ fase, seguidos pelos da $10^{\mathrm{a}}$ fase, enquanto poucos estudantes da $1^{\mathrm{a}}$ fase consideraram as condições ruins/péssimas, tendendo a tê-las considerado regulares. Isso provavelmente pode ser explicado pelo fato de os estudantes da $1^{\text {a }}$ fase terem majoritariamente aulas teóricas em vários centros do campus e pouca quantidade de aula prática, quando comparados às demais fases. A $5^{\mathrm{a}}$ fase tem quantidade significativa de aula prática préclínica no prédio do curso de Odontologia e a $10^{\mathrm{a}}$ fase tem quase que somente prática clínica no mesmo local.
O pensamento de desistir do curso ocorre pouco na $1^{\mathrm{a}}$ fase e se apresenta mais elevado na $5^{\mathrm{a}}$ e na $10^{\mathrm{a}}$ fase, em proporções semelhantes, com associação estatisticamente significativa.

Nota-se que, apesar de compor a maior parte da amostra, nenhum estudante da $1^{a}$ fase do curso é acometido pela SB. Isso pode sugerir que os estudantes chegam à universidade sem SB. O período em que o Burnout é mais prevalente é na $5^{\text {a }}$ fase, a qual possui a carga horária mais pesada da graduação de Odontologia na UFSC, deixando livre apenas meio turno por semana. Garbin et al. (2012) apresentaram correlação significativa entre o número de disciplinas em que se está matriculado no Curso de Graduação em Odontologia da UNESP Araçatuba e a dimensão EE. ${ }^{13}$ Isso ocorre, segundo o autor, devido à carga demasiada de conhecimento teórico e à ocorrência de muitas provas. ${ }^{13} \mathrm{~A} 10^{\mathrm{a}}$ fase também tem uma 
parcela de estudantes com SB, o que pode ser resultado do esgotamento físico e mental do final de curso e da pressão para o ingresso no mercado de trabalho.
Na Tabela 2 encontra-se o estudo de associação entre o perfil dos estudantes e a ocorrência de SB.

Tabela 2 - Estudo de associação entre perfil dos estudantes e ocorrência da síndrome de Burnout (quando $\mathrm{p} \leq 0,05$ ). Curso de graduação em Odontologia. UFSC. Florianópolis-SC, 2013.

\begin{tabular}{|c|c|c|c|}
\hline Característica & \multicolumn{2}{|c|}{ Ocorrência da síndrome de Burnout $(\mathrm{n}(\%))$} & \multirow[t]{2}{*}{$\mathbf{p}$} \\
\hline Horas semanais de lazer & Não & Sim & \\
\hline Nenhuma & $1(50)$ & $1(50)$ & \\
\hline Entre 1 e 5 horas & $43(84,31)$ & $8(15,69)$ & \\
\hline Mais de 5 horas & $46(95,83)$ & $2(4,17)$ & $\mathbf{0 , 0 3 6}$ \\
\hline \multicolumn{4}{|l|}{ Horas diárias de sono } \\
\hline Menos de 6 horas & $28(80)$ & $7(20)$ & \\
\hline Entre 6 e 8 horas & $62(93,94)$ & $4(6,06)$ & 0,045 \\
\hline \multicolumn{4}{|l|}{$\begin{array}{l}\text { Necessidade de professor } \\
\text { particular }\end{array}$} \\
\hline Não & $89(90,82)$ & $9(9,18)$ & \\
\hline Sim & $1(33,33)$ & $2(66,67)$ & $\mathbf{0 , 0 3 1}$ \\
\hline \multicolumn{4}{|c|}{ Autopercepção desempenho acadêmico } \\
\hline Ruim/Péssimo & $4(80)$ & $1(20)$ & \\
\hline Regular & $22(70,97)$ & $9(29,03)$ & \\
\hline Bom/Excelente & $64(98,46)$ & $1(1,54)$ & 0,000 \\
\hline \multicolumn{4}{|c|}{ Percepção desempenho professores } \\
\hline Ruim/Péssimo & $1(50)$ & $1(50)$ & \\
\hline Regular & $24(77,42)$ & $7(22,58)$ & \\
\hline Bom/Excelente & $65(95,59)$ & $3(4,41)$ & $\mathbf{0 , 0 1 1}$ \\
\hline \multicolumn{4}{|c|}{ Percepção das condições da estrutura física } \\
\hline Ruins/Péssimas & $36(78,26)$ & $10(21,74)$ & \\
\hline Regulares & $5(97,22)$ & $1(2,78)$ & \\
\hline Boas/Excelentes & $19(100)$ & $-(-)$ & $\mathbf{0 , 0 1 1}$ \\
\hline \multicolumn{4}{|c|}{ Consome medicamentos devido aos estudos } \\
\hline Não & $63(94,03)$ & $4(5,97)$ & \\
\hline Sim & $27(79,41)$ & $7(20,59)$ & $\mathbf{0 , 0 4 0}$ \\
\hline \multicolumn{4}{|c|}{ Já pensou em desistir do curso } \\
\hline Não & $55(94,83)$ & $3(5,17)$ & \\
\hline Sim & $35(81,4)$ & $8(18,6)$ & 0,050 \\
\hline
\end{tabular}

Houve associação estatisticamente significativa entre SB e as variáveis: horas semanais de lazer $(\mathrm{p}<0,05)$, horas diárias de sono $(\mathrm{p}<0,05)$, necessidade de professor particular $\quad(p<0,05), \quad$ autopercepção do desempenho acadêmico $(\mathrm{p}<0,01)$, percepção do desempenho dos professores $(\mathrm{p}<0,05)$ e das condições da estrutura física da instituição de ensino $(p<0,05)$, consumo de medicamentos devido aos estudos $(\mathrm{p}<0,05)$ e pensamento de desistir do curso $(\mathrm{p} \leq 0,05)$. Para associação com a SB, os estudantes que pensam às vezes e com frequência em desistir do curso foram agrupados em um único grupo, o dos que já pensaram em desistir do curso.

Os estudantes que dedicaram mais de 5 horas semanais para o lazer, os que dormiram entre 6 e 8 horas por dia, os que consideraram seu desempenho acadêmico, o 
dos professores e a estrutura física da instituição de ensino bons/excelentes, os que não consumiam medicamentos devido aos estudos e não pensaram em desistir do curso foram os menos acometidos pela SB. Por outro lado, os estudantes que precisaram de professor particular foram os mais acometidos por este agravo.

Corroborando com esse estudo, Campos et al. (2012), também notaram que estudantes com desempenho acadêmico ruim, que consomem medicação devido aos estudos e que já pensaram em desistir do curso foram mais acometidos pela SB do que os demais. ${ }^{4}$

Estes pesquisadores também sugerem que o desempenho acadêmico ruim associado à SB pode ocorrer porque as atividades acadêmicas se tornam uma tarefa mais desgastante para os estudantes com SB, o que induz à descrença (DE). ${ }^{4}$ Quanto à ingestão de medicamentos devido aos estudos, os autores sugerem que esta possa ser uma consequência do aparecimento da SB, mas salientam que essa informação deve ser interpretada com cuidado, já que os estudos são de corte transversal de natureza correlacional. ${ }^{4}$ Para Visoso, Sánchez e Montiel (2012), o consumo de medicamentos e o desejo de abandonar o trabalho ou o estudo podem ocorrer como uma consequência adversa da SB. ${ }^{14}$

Batista et al. (2010), ao estudarem a SB em professores, sugerem que a intenção de desistir do trabalho pode ser considerada uma tentativa de lidar com a exaustão, que muitas vezes é advinda como resultado de recompensas inferiores aos investimentos e esforços realizados. Da mesma forma pode-se considerar com o estudante, quando este pensa em desistir do curso. ${ }^{27}$

O National Institute for Ocupational Safety and Health (NIOSH, 2002), sugere que as condições de trabalho podem interferir na produtividade do trabalhador e contribuir para o desenvolvimento de estresse ocupacional. ${ }^{28}$ Se aplicarmos essa hipótese para a realidade dos estudantes de Odontologia, é possível entender porque os universitários que, nesse estudo, consideram a estrutura física de sua instituição de ensino péssima são os mais acometidos pela SB. A precariedade das condições de trabalho levam à insatisfação pessoal e à queda na produtividade. ${ }^{26}$

\section{CONCLUSÃO}

A prevalência da SB na amostra foi de 10,89\% (IC95\% = 4,71 - 17,07). Os estudantes de graduação em Odontologia são acometidos pela síndrome de Burnout, que está associada à fase do curso, tempo de lazer e de sono, necessidade de professor particular, percepções frente ao ambiente acadêmico, consumo de medicamentos devido aos estudos e pensamento de desistir do curso.

\section{ABSTRACT \\ Burnout in undergraduate dental students}

The Burnout syndrome (BS) is a multidimensional chronic condition. It is observed that a significant number of health professionals and students, especially on Dentistry, has been affected by this injury. This study aimed to check the prevalence of SB among undergraduate dental students of 1st, 5th and 10th stages of the Federal University of Santa Catarina (UFSC). The instrument for data collection was a questionnaire on demographic and economic data, student profile and the Maslach Burnout Inventory - Student Survey (MBI-SS) in the Brazilian version. The prevalence of SB was $10.89 \%$. There was a statistically significant association between the stages of the course and the variables perception of the conditions of the physical structure of the educational institution and thought of quitting the course. Also between the occurrence of the SB and the variables: stage of the course, weekly hours of 
leisure, time spent sleeping, need a tutor, selfperception of the academic performance and of the teacher's one, perception of the conditions of the physical structure of the educational institution, consumption medicines because studies and thought of quitting the course. In conclusion, the SB was prevalent in $10.89 \%$ of the students surveyed.

Descriptors: Burnout. Stress. Dental Students.

\section{REFERÊNCIAS}

1. Costa JRAD, Lima JVD, Almeida PCD. Stress no trabalho do enfermeiro. Rev Esc Enf USP. 2003 [acessado em 1 mar 2016]; 37(3). Disponível em: http://www. scielo.br/pdf/reeusp/v37n3/08.pdf

2. Maslach C, Jackson SE. The measurement of experienced burnout. J Organ Behav. 1981; [cited 2013 Jun 17]; 2(2). Available from: http://doi.wiley.com/10.1002/job.40 30020205

3. Ribeiro SS, Motta EAP. Associação entre a síndrome de burnout e o hormônio cortisol. Rev Ciênc Saúde. 2014 [acessado em 1 mar 2016]; 16(2). Disponível em: http://www.periodicoseletronicos.ufma.br/ index.php/rcisaude/article/view/4081/216 $\underline{4}$

4. Campos JADB, Jordani PC, Zucoloto ML, Bonafé FSS, Maroco J. Síndrome de Burnout em graduandos de Odontologia. Rev Bras Epidemiol. 2012 [acessado em 17 jun 2013];15(1). Disponível em: http:// www.scielo.br/pdf/rbepid/v15n1/14.pdf

5. Birks Y, McKendree J, Watt I. Emotional intelligence and perceived stress in healthcare students: a multi-institutional, multi-professional survey. BMC Med Educ. 2009 [cited 2013 Jun 17]; 9(1). Available from: http://bmcmededuc. biomedcentral.com/articles/10.1186/1472$\underline{6920-9-61}$
6. Martínez AA, Aytés LB, Escoda CG. The burnout syndrome and associated personality disturbances. The study in three graduate programs in Dentistry at the University of Barcelona. Med Oral Patol Oral Cir Bucal. 2008 [cited 2013 Jun 17];13(7): Available from: http://diposit. ub.edu/dspace/handle/2445/48468

7. Moysés ST, Moysés SJ, Kriger L, Schmitt EJ. Humanizando a educação em Odontologia. Rev ABENO. 2003 [acessado em 17 jun 2013];3(1): Disponível em: http://www.universidade saudavel.com.br/wpcontent/uploads/ARTI GOS/artigo 1 ABENO.pdf

8. Lemos CLS. A implantação das Diretrizes Curriculares dos Cursos de Graduação em Odontologia no Brasil: algumas reflexões. Rev ABENO. 2004 [acessado em 17 jun 2013];1(5): Disponível em: http://www. abeno.org.br/aadm/adm/revista/arquivos pdf/2005/Abeno_5-1.pdf

9. Gorter R, Freeman R, Hammen S, Murtomaa H, Blinkhorn A, Humphris G. Psychological stress and health in undergraduate dental students: fifth year outcomes compared with first year baseline results from five European dental schools. Eur J Dent Educ. 2008 [cited 2013 Jun 17];12 Available from: http://onlinelibrary. wiley.com/doi/10.1111/j.1600-0579. 2008 $.00468 . x / p d f$

10. Martínez AA, Aytés LB, Escoda CG. The burnout syndrome and associated personality disturbances. The study in three graduate programs in Dentistry at the University of Barcelona. Med Oral Patol Oral Cir Bucal. 2008;13(7):444-50.

11. Preciado-Serrano ML, Vázquez-Goñi JM. Perfil de estrés y síndrome de burnout en estudiantes mexicanos de odontologia de una universidad pública. Rev Chil Neuro- 
Psiquiat 2010 [cited 2013 Jun 17]; 48(1). Available from: http://www.scielo.cl/pdf/ rchnp/v48n1/art02.pdf.

12. Divaris K, Lai CS, Polychronopoulou A, Eliades T, Katsaros C. Stress and burnout among Swiss dental residents. Schweiz Monatsschr Zahnmed. 2012;122:610-5.

13. Garbin CAS, Saliba NA, Santos RR dos, Prado RL do, Garbin AJI. Burnout en estudiantes de odontología: evaluación a través MBI - versión. Med Segur Trab. 2012;58(229):327-34.

14. Visoso SA, Sánchez RPA, Montiel BNM. Síndrome de Burnout en la Facultad de Odontología de la Universidad Autónoma del estado de México: un estudio comparativo. Int J Odontostomat 2012 [cited 2013 Jun 17];6(2). Available from: http://www.scielo.cl/pdf/ijodontos/v6n2/ar t03.pdf

15. Associação Brasileira de Empresas de Pesquisa (ABEP). Critério de Classificação Econômica Brasil. São Paulo: 2013 [acessado em 3 abr 2014]. Disponível em: http://www.abep.org/novo /FileGene rate. $\operatorname{ash} x$ ? $\mathrm{id}=296$.

16. Campos JADB, Zucoloto ML, Bonafé FSS, Jordani PC, Maroco J. Reliability and validity of self-reported burnout in college students: A cross randomized comparison of paper-and-pencil vs. online administration. Comput Human Behav. 2011 Sep [cited 2013 Jun 12];27(5). Available from: http:// linkinghub. elsevier.com/retrieve/pii/S0747563211000 $\underline{77 X}$

17. Maslach C, Jackson SE. Maslach burnout inventory manual. Palo Alto, CA: Consulting Psychologists Press; 1986.

18. Montero-Marin J, Monticelli F, Casas M, Roman A, Tomas I, Gili M, GarciaCampayo J. Burnout syndrome among dental students: a short version of the "Burnout Clinical Subtype Questionnaire" adapted for students (BCSQ-12-SS). BMC Med Educ 2011; [cited 2013 Jun 17];11(1). Available from: http://www.biomed central.com/1472-6920/11/103.

19. Carson E, Fearnley S. Careers of south Australian health professional graduates. Aust Bull Labour. 2010;36(1):1-28.

20. Gietzelt D. Social profile of first-year dentistry students at the University of Sydney. Aust Dent J 1997 [cited 2014 Feb 25];42(4). Available from: http://www. ncbi. nlm.nih.gov/pubmed/9316314

21. Schmitt A, Rosenfeldt YAZ, Oliveira MO, Rosolem GPN, Loch C. Proposta de mobilidade coletiva para a Região Metropolitana de Florianópolis pensada a partir de imagens do Satélite [Internet]. In: Anais do XVI Simpósio Brasileiro de Sensoriamento Remoto - SBSR; 2013 [acessado em 2 abr 2014]; Foz do Iguaçu. Disponível em: http://www.dsr.inpe.br/ sbsr2013/files/p1352.pdf

22. Prinz P, Hertrich K, Hirschfelder U, Zwaan $M$ de. Burnout, depression and depersonalisation-Psychological factors and coping strategies in dental and medical students. GMS Z Med Ausbild. 2012 [cited 2013 Jun 17];29(1). Available from: http://www.ncbi.nlm.nih.gov/pmc/articles/ PMC 3296106/pdf/ZMA-29-10.pdf

23. Michal M. Stress, sinais e causas; v. 1. São Paulo: Roche; 1997, 40 p.

24. Lemos CLS. Saberes e práticas curriculares: um estudo do curso de Odontologia da Universidade Federal de Uberlândia [dissertação]. Uberlândia MG): Universidade Federal de Uberlândia; 2003. 25. Fernandes RMF. O sono normal [Internet]. In: Simpósio Distúrbios Respiratórios do Sono. Ribeirão Preto; 
2006 [acessado 2014 Abr 2]:9(2):[ Disponível em: http://www.revistas .usp.br/rmrp/article/download/372/373

26. Regis Filho GI, Ribeiro DM. Estresse e Qualidade de Vida no Trabalho do Cirurgião-Dentista. Florianópolis: Insular, 2007. 113 p.

27. Batista JBV, Carlotto MS, Coutinho AS, Augusto LG da S. Prevalência da síndrome de Burnout e fatores sociodemográficos e laborais em professores de escolas municipais da cidade de João Pessoa, PB. Rev Bras Epidemiol [periódico na Internet] 2010 [acessado 2014 Mar 11];13(3): Disponível em: http://www.scielo.br/ pdf/rbepid/v13n3/13.pdf
28. National Institute for Occupational Safety And Health. Stress at work. U.S. Department of Health and Human Services. US Government Printing Office, Washington, DC, 2002; 99(1001).

Correspondência para:

Camila Prado das Neves

e-mail: camilaneves32@gmail.com

Rua Lauro Linhares, n. ${ }^{\circ}$ 1830, apto. 401, bloco Argentina, bairro Trindade. Florianópolis-SC. 88036-002. 